\title{
Direct Laser Writing of Functional Optofluidic Elements in Porous Silicate Matrix
}

\author{
Roman A. Zakoldaev*1, Zhong Lijing ${ }^{2}$, Veronika A. Yakimuk ${ }^{1}$, Yulia I. Yandybaeva ${ }^{1}$, Alena S. Shishkina ${ }^{1}$, and \\ Olga V. Andreeva ${ }^{1}$
}

\author{
${ }^{1}$ ITMO University, 49, Kronverkskiy av., 197101, St. Petersburg, Russia \\ ${ }^{2}$ Zhejiang Lab, Hangzhou City, Zhejiang Province, 311121, P.R. China \\ "Corresponding author's e-mail: zakoldaev@gmail.com
}

\begin{abstract}
The integration of optical, micro-, and nanofluid structures in glass has been always relevant for sensoric and analytical tasks performed on a single chip. Direct laser writing (DLW) makes the integration step fast providing required functional elements with high precision and unique structural features. Here, we consider a porous silicate matrix (PSM) as a base for the optofluidic elements and their type expansion. Three types of elements are presented - optical waveguides, barriers, and microsized hollow channels. All these elements are fabricated by changing the density of the PSM by tuning laser processing parameters. The work reveals new experimental study for each element - the formation and investigation of symmetrical optical waveguides, optimization of the pass-through barriers writing, microchannel fabrication.
\end{abstract}

DOI: 10.2961/jlmn.2021.03.2001

Keywords: porous silicate matrix, DLW, optofluidic, waveguides, microfluidic channel, barriers

\section{Introduction}

Direct laser writing (DLW) in glass is gaining popularity to integrate functional elements such as waveguides [1], hollow channels [2], and microcavity [3] inside a glass substrate. This trend is also justified by the development of integrated optics, sensorics, and chip-scale devices. Solid and single-component glass, such as fused silica, is a classical base for opto-fluid [4] and micromechanical systems [5]. On the other hand, the initial density of glass restricts the thresholds for laser processing, limiting the variation in the properties of optofluidic elements.

Porous silicate matrices (PSM) can significantly expand the functionality of the elements and offer a novel architecture. Noteworthy, PSM is also produced in the form of optical blanks with the required size and thickness. Previous studies on laser-induced modifications of porous glass [6], sol-gel, and aerogel [7] have confirmed the possibility to vary the material density. That opened the way for DLW of waveguides [8], integral gas-analytical cells [9], microfluidics [10], and nanofluidic [11] channels. The laserinduced change is expressed as nanopore closure, nanogratting formation, or material foaming. The difference in the composition of the original glass blanks significantly complicates the obtaining of stable and identical results. Therefore, we have initiated a research project to develop methods for the femtosecond DLW in PSM produced from one glass melting batch.

Herein, we present the results of recording three elements in PSM by changing focusing conditions and laser processing parameters. Among these elements are (i) waveguides with almost symmetrical shape with the refractive index contrast $1.54 \cdot 10^{-3}$; (ii) the barriers isolating the PSM plate; (iii) micro-channels in PSM without chemical etching and sintering. Remarkably, all the investigations were accomplished inside PSM with through pores and large free pore volume $(\sim 50 \%)$ promising great opportunities for modifying the sample structure under the action of laser radiation.

\section{Experiment and Methods}

The samples are produced from two-phase glass providing stable and reproducible characteristics. In particular, our PSM-17 possesses thickness $1.0 \pm 0.1 \mathrm{~mm}$, pore size $\sim 17 \mathrm{~nm}$, and porosity 50-54\% [12]. The sample consists of $>90 \% \mathrm{SiO}_{2}$ including an insignificant amount of impurities in the form of $\mathrm{B}_{2} \mathrm{O}_{3}, \mathrm{Na}_{2} \mathrm{O}$. The photos and spectral characterization show high uniformity and optical transparency higher than $80 \%$ in the range of $600-1100 \mathrm{~nm}$ (Fig. 1).
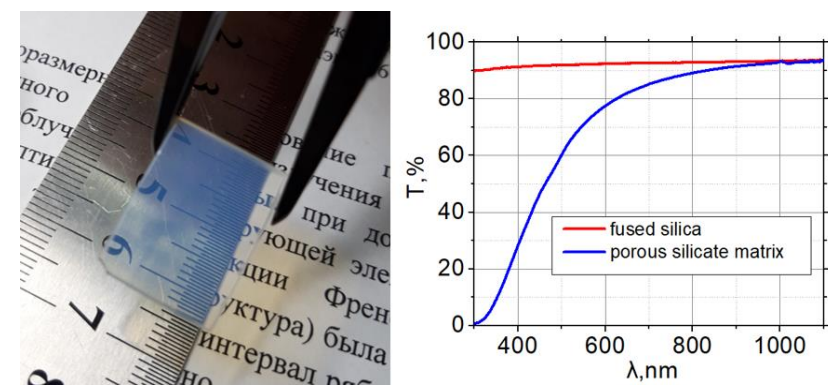

Fig.1. Photo of PSM-17 sample with a size of $15 \times 20 \times 1 \mathrm{~mm}$ and transmission spectrum in the range $200-1100 \mathrm{~nm}$ with the reference to fused silica.

Laser writing was performed on an experimental setup (Fig. 2) with an ytterbium fiber femtosecond laser (ANTAUS-20W-20u/1M, Avesta Ltd., Moscow, Russia) as a radiation source. The laser generates pulses with duration $0.22-3.0 \mathrm{ps}$ and frequency up to $1 \mathrm{MHz}$. At the output of 
the system, the average power is $\mathrm{P}_{0}=20 \mathrm{~W}$ for wavelength $1030 \mathrm{~nm}$. Table 1 presents the information about the lenses applied. The choice of a lens is discussed in the following section. The sample was fixed and translated on a programmable coordinate system with minimum step 500 $\mathrm{nm}$ and maximum speed up to $100 \mathrm{~mm} / \mathrm{s}$.

Table 1 - List of applied objectives

\begin{tabular}{cc}
\multicolumn{2}{c}{ Table 1 - List of applied objectives } \\
\hline Title & Task \\
\hline 60X, NA $=0.85$ & waveguides \\
20X, NA $=0.4$ & channels \\
Plano convex lens, $\mathrm{F}=200 \mathrm{~mm}$ & barriers \\
\hline
\end{tabular}

Microphoto images of the final structures were captured by a microscope (Axio Imager A1m, Carl Zeiss). The sample ends were ground and polished by a polishing unit (Buehler MetaServ 250). Laser radiation coupling by the objective (60X, NA=0.85) - objective (20X, NA=0.4) connection was organized to test the fabricated waveguides and capture near-field intensity distribution.

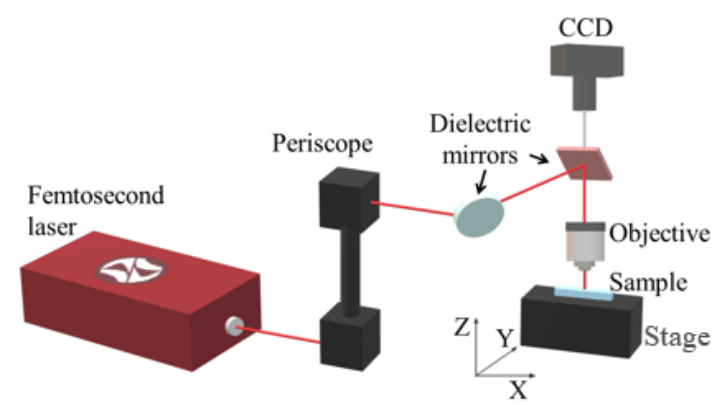

Fig.2. Schematic illustration of the experimental setup for DLW in PSM.

\section{Results and discussion}

This study demonstrates the fabrication of the waveguides, impermeable barriers, and microchannels in the PSM plate. In the following subsections, we focus on each element separately.

\subsection{Waveguides writing}

Waveguides are fabricated with sharp focusing of laser radiation using the objective with $60 \mathrm{X}$ magnification (Table 1). The formation of elongated regions with a large aspect ratio is a known obstacle in the field of waveguide writing. An elementary solution is the use of the slit technique, which is described in detail in [13]. Briefly, the slit beam shaping technique operates by placing the slit directly in front of the objective. The slit size can be calculated by the following equation:

$$
\frac{W_{y}}{W_{x}}=\frac{N A}{n} \sqrt{\frac{\ln 2}{3}}, \text { if } W_{x}>3 W_{y},(1)
$$

where $W_{y}$ and $W_{x}$ are the slit width, $N A \sim f / W_{x}$ where $f$ is the focal length of the objective, and $n$ is the refractive index of PSM. This technique has never been used for DLW of waveguides in PSM before.

Figures $3 \mathrm{a}$ and $3 \mathrm{~b}$ demonstrate the top view and crosssectional micro photos of the fabricated waveguides buried at $250 \mu \mathrm{m}$ inside the sample. When a slit $(\sim 1.0 \mathrm{~mm})$ was utilized, the average longitudinal and transverse sizes were $3.0 \mu \mathrm{m}$ and $2.8 \mu \mathrm{m}$, respectively. The aspect ratio of the manufactured waveguides is 1.07 . We also determined the minimum period $(p)$ for waveguides arrangement. When we fabricated closed packed waveguides with period of displacement $\mathrm{p}<5 \mathrm{um}$, we noticed the reshaping of the previously fabricated waveguide. As a result, the aspect ratio is changed (Fig. 3, b, set with $p=3$ ). If we increase $p>5$, the fabrication process does not influence the previously fabricated waveguide. Such a small period resulted in no defects across the waveguides, which is one more benefit of the technique applied.

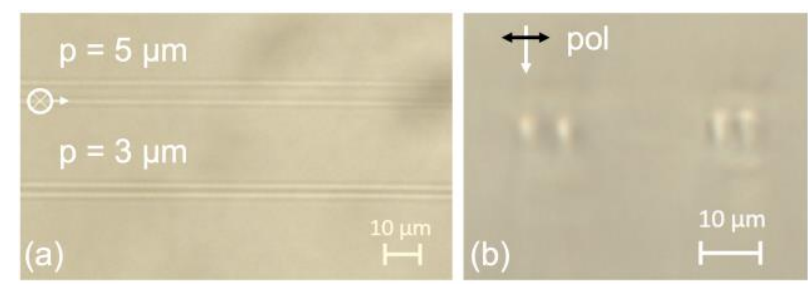

Fig.3. Micro photos of waveguides buried at $250 \mu \mathrm{m}$ inside PSM: (a) top view and (b) cross-section. Writing parameters: $\mathrm{P}_{0}=2 \mathrm{~W}$, scanning speed $45 \mathrm{~mm} / \mathrm{s}$. The laser beam direction is indicated by the white arrow. The radiation polarization is indicated by black arrow.

The cross-sectional photo shows a bright almost circular spot resulting from the increment in the refractive index. On the left (Fig. 3, b), under the bright spot, there is a subtle dark area that accompanies all the created waveguides. The appearance of the dark region is the result of light scattering that occurs on the decompressed zone of the waveguide. Optical microscopy does not allow to see in detail such a decompressed region. The refractive index contrast $(\Delta n)$ in this cross-section differs compared to the initial material and the waveguide core. For this, the near field distribution of the He-Ne laser radiation was captured (Fig. 4, a). The observed modes have a good degree of symmetry and beam diameter $\sim 4 \mu \mathrm{m}$. The data obtained also allows to evaluate the refractive index contrast by the method applied earlier [14]. The calculations made it possible to estimate the contrast, $\Delta \mathrm{n}=1.54 \cdot 10^{-3}$ (Fig. $4, \mathrm{~b}$ ).
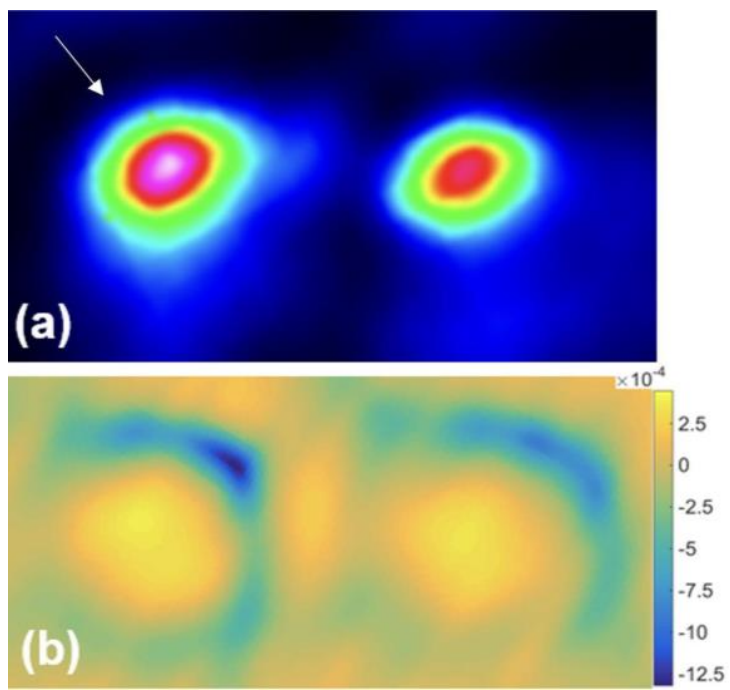

Fig.4. The near field image of guided modes at $635 \mathrm{~nm}$ for two waveguides located with $p=9 \mu \mathrm{m}$ (a). Estimated refractive index contrast profile (b).

The He-Ne laser beam was coupled into the left waveguide (indicated by an arrow in Fig. 4, a) through an 
objective (60X, $0.85 \mathrm{NA})$, and its output beam was collected by another objective (40X, $0.65 \mathrm{NA})$. At the output side of the dual-waveguides, effective light coupling into the right waveguide was observed, the intensity ratio between the left and the right waveguide is $\sim 5: 4$. This phenomenon is attributed to the crosstalk between the dual waveguides due to the overlap of their cladding or evanescent wave field [15].

\subsection{Impenetrable barriers}

The initial PSM has a nanoporous framework, which we modify by DLW. Thus, the fabricated uniform tracks can collapse the nanopores locally to control their throughput and hence the flow of nanoobjects trapped by pores. We called these tracks barriers aimed at holding the flow of nanoobjects inside the glass. Previously, we demonstrated the isolation of several independent zones in a single PSM for sensing purposes [16]. However, every barrier consisted of several densified tracks providing full-thickness filling of the glass plate. This time, we optimized the procedure to fabricate the barrier by a single scan. The radiation is now focused by the lens (Table 1) with a long focal length and a larger Rayleigh zone compared to the objectives.

First, we optimized the laser power to write homogeneous barriers. At a constant speed $(1 \mathrm{~mm} / \mathrm{s})$, a set of tracks with different power (1200-1600 mW) was fabricated at a frequency of $200 \mathrm{kHz}$. The set fragment is shown in figure 5 , a. The average width is $5 \mu \mathrm{m}$ and the irregularities achieved along every track. That is mostly the result of external vibrations. Changing the scanning speed in the range of $0.5-20 \mathrm{~mm} / \mathrm{s}$ made it possible to determine the optimal scanning speed to obtain a uniform barrier for speed $>5 \mathrm{~mm} / \mathrm{s}$.

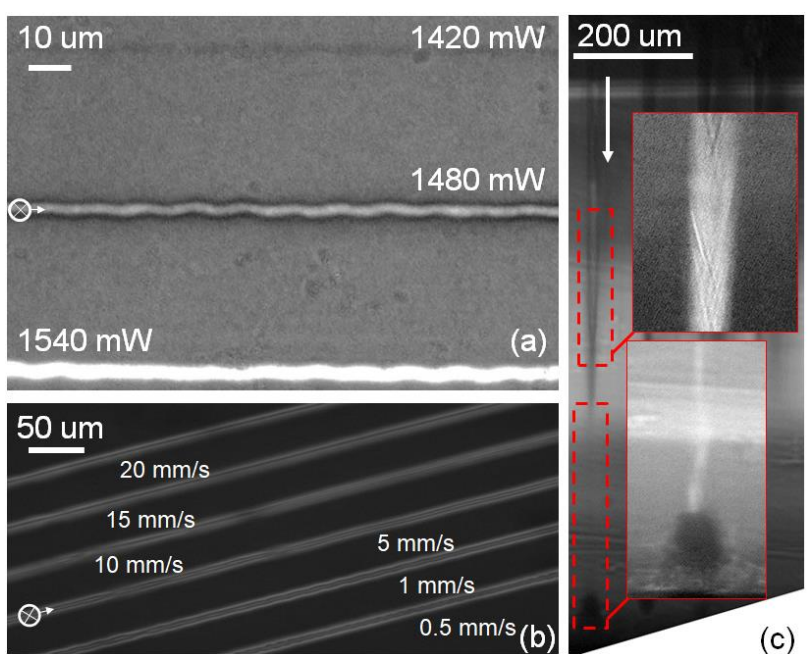

Fig.5. Micro photos of barriers fabricated by DLW in PSM with constant scanning speed $(1 \mathrm{~mm} / \mathrm{s})$ and different power $(1540$, 1490 and $1420 \mathrm{~mW}$ ) (a). The influence of scanning speed (0.5 $20 \mathrm{~mm} / \mathrm{s}$ ) on the uniform shape of the barrier at constant power

$(1540 \mathrm{~mW})(\mathrm{b})$. Cross-section of the barrier, which length corresponds to the glass thickness (c). The laser beam direction is indicated by the white arrow.

The barrier depth is an important characteristic, and in our case, the minimum value was $400 \mu \mathrm{m}$ at the minimum laser radiation power $(1200 \mathrm{~mW})$. By increasing the power to $1540 \mathrm{~mW}$ at the speed of $10 \mathrm{~mm} / \mathrm{s}$, it was possible to fabricate a track with the depth commensurate with the thickness of PSM $\sim 1 \mathrm{~mm}$ (Fig. 5, c). In cross-section, the barrier does not remain constant in size. As a result of selffocusing [17], we observe its broadening to $20 \mu \mathrm{m}$ in the upper part, and narrowing to $5 \mu \mathrm{m}$ from the bottom (Fig. 5, c). On the lower surface of the glass, a local decompaction of the material in the form of a dark sphere is also noticeable.

\subsection{Hollow micro-channels}

A gradual increase in the power of the laser radiation led to the formation of highly scattering tracks attributed to the local glass destruction. Previously, it was shown microchambers and channels fabrication by simultaneous controlled laser-induced destruction of nanoporous materials with subsequent thermal sintering [18]. The centimeter channels with square cross-section were formed beneath the glass surface by a femtosecond laser writing inside a water-immersed sample.

Our current research aims to fabricate channels and save the nanoporous structure without chemical etching. First, the channel prototype was formed by using a lower frequency, $10 \mathrm{~Hz}$, and a higher pulse energy, $100-200 \mu \mathrm{J}$, with pulse duration 30 ps (Fig. 6, a). The uniform brightness of the structure under a crossed polarizer and analyzer indicates the presence of debris across the track (Fig. 6, b). The crosssection of the channel is $53 \times 16 \mu \mathrm{m}$ (Fig. 6, c).

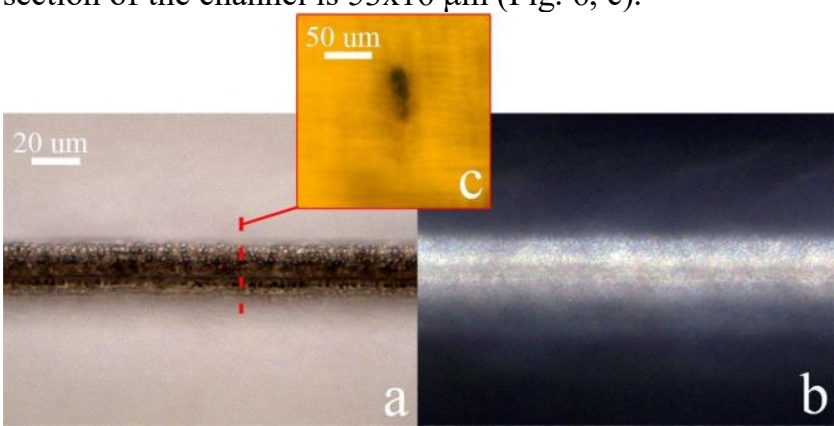

Fig.6. Microphotos of channel prototype fabricated with picosecond laser $(335 \mathrm{~nm}, 30 \mathrm{ps}, 200 \mu \mathrm{J})$ in transmitted (a) and cross-polarized light (b) The channel cross-section (c).

The debris was removed by ultrasonic cleaning in water. The action of ultrasound ( $5 \mathrm{~min}$, temperature $31^{\circ} \mathrm{C}$ ) led to a noticeable clarification of the track (Fig. 7). It can be assumed that the nanoporous framework undergoes the action of ultrasound and promotes the removal of debris. The measurement of the channel cleaning speed was performed after 5 minutes and consisted of measuring the length of the cleaned area when examining the sample with an optical microscope. The presence of uncleaned areas is noticeable in the crossed polarizer and analyzer pair. The data obtained allowed to determine the cleaning speed. The length, $300 \mu \mathrm{m}$, of microchannel was cleaned at the maximum speed (60 $\mu \mathrm{m} / \mathrm{min}$ ), while the subsequent sections passed the cleaning stage at a lower speed, up to $40 \mu \mathrm{m} / \mathrm{min}$. Thus, the average speed of cleaning a channel with the length of $800 \mu \mathrm{m}$ equaled $\sim 50 \mu \mathrm{m} / \mathrm{min}$. The decrease in the cleaning speed is due to a slowdown in the inflow of water and the outflow of removal products from the channel. 


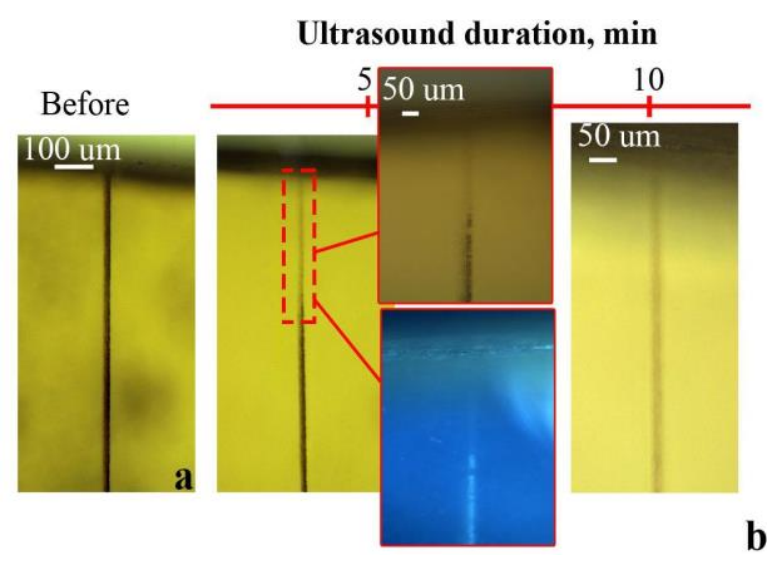

Fig.7. Microphoto of channel prototype before (a) and after ultrasound cleaning in water (b) after 5 and 10 minutes.

Based on the literature review [19], we can assume that longer channels are more complicated to clean. Debris can block the water flow. However, several ways can be used to overcome this obstacle - channel widening and multi-pass writing. More complex way is to create additional channels with output to the surface [4].

At the next step, we decided to increase the speed of through channels writing and applied higher frequency with help of fs-laser setup. Changing the objective from $20 \mathrm{X}$ to $60 \mathrm{X}$ allowed us to reduce the waist diameter, as well as the channel cross-section $(9 \times 23 \mu \mathrm{m})$. The frequency, up to 200 $\mathrm{kHz}$, was applied to avoid the consolidation of derbes that was discussed elsewhere [20]. Then, the set of channels prototypes was fabricated with constant laser power, 740 $\mathrm{mW}$, and frequency - $200 \mathrm{kHz}$ (Fig. 8, a). Then, we cleaned channels under the action of ultrasound with the parameters: $150 \mathrm{~min}, 31^{\circ} \mathrm{C}$ (Fig. 8, b).

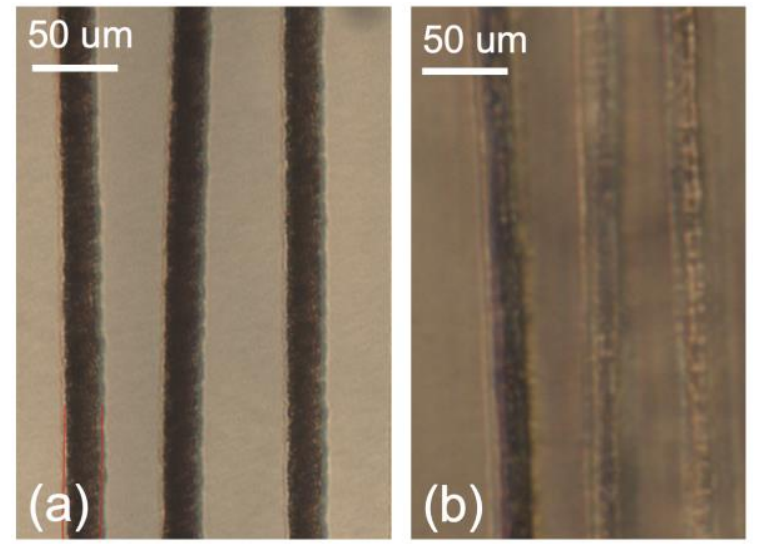

Fig.8. Micro photos of channel prototype set fabricated inside PSM, $\mathrm{P}_{0}=740 \mathrm{~mW}$, scanning speed $45 \mathrm{~mm} / \mathrm{s}$, objective $60 \mathrm{X}$, $\mathrm{NA}=0,85, v=200 \mathrm{kHz},:$ (a) after laser direct writing and (b) after cleaning in the water under ultrasound action.

\section{Conclusion}

Applying the direct laser writing technique we have fabricated three optofluidic elements inside PSM. The symmetrical (aspect ratio 1.07) waveguide was fabricated at high scanning speed $45 \mathrm{~mm} / \mathrm{s}$. The minimum period of waveguide placement was determined, $p \geq 5 \mu \mathrm{m}$. If the period is decreased, $p=3 \mu \mathrm{m}$, the aspect ratio of the waveguides changes.
We have demonstrated the fabrication of pass-through barriers by a single scan. The use of a long-focus lens made it possible to collapse the nanoporous framework locally over the PSM sample thickness.

Microchannel prototypes were successfully fabricated in the PSM, and then cleaned in water under the action of ultrasound. The nanoporous structure remained preserved as chemical etching and complete sintering of PSM was not applied. The average speed of cleaning a channel with the length of $800 \mu \mathrm{m}$ equaled $\sim 50 \mu \mathrm{m} / \mathrm{min}$.

\section{Acknowledgments}

The study is funded by the grant of Russian Science Foundation (project № 20-71-10103).

\section{References}

[1] M. Macias-Montero, F. Muñoz, B. Sotillo, J. del Hoyo, R. Ariza, P. Fernandez, J. Siegel, and J. Solis: Sci. Rep., 11, (2021) 8390.

[2] Xiaolong Li, Jian Xu, Zijie Lin, Jia Qi, Peng Wang, Wei Chu, Zhiwei Fang, Zhenhua Wang, Zhifang Chai, Ya Cheng: Appl. Surf. Sci., 485, (2019) 188.

[3] S.S. Fedotov, A.S. Lipatiev, M.Y. Presniakov, G.Y. Shakhgildyan, A.G. Okhrimchuk, S.V. Lotarev, and V.N. Sigaev: Opt. Lett., 45, (2020) 5424.

[4] Zhengming Liu, Jian Xu, Zijie Lin, Jia Qi, Xiaolong Li, Aodong Zhang, Jintian Lin, Jianfang Chen, Zhiwei Fang, Yunpeng Song, Wei Chu, Ya Cheng: Opt. Laser Technol., 141, (2021) 107118.

[5] V. Stankevic, T. Rakickas, and G. Raciukaitis: J. Laser Micro Nanoeng., 11, (2016) 53.

[6] L. Zhong, R. A. Zakoldaev, M. M. Sergeev, V. P. Veiko, and Z. Li: Opt. Quantum Electron., 49, (2020) 1

[7] A. Cerkauskaite, R. Drevinskas, A.O. Rybaltovskii, and P.G. Kazansky: Opt. Express, 25, (2017) 8011.

[8] J.V. Mikhailova, L. Zhong, A.A. Ostanin, M.M. Sergeev, R.A. Zakoldaev: J. Laser Micro Nanoeng., 15, (2020) 252. [9] V.P. Veiko, R.A. Zakoldaev, M.M. Sergeev, P.A. Danilov, S.I. Kudryashov, G.K. Kostiuk, A.N. Sivers, A.A. Ionin, T.V. Antropova, and O.S. Medvedev: Opt. Express, 26, (2018) 28150.

[10] K. Liao, W. Wang, X. Mei and B. Liu: Opt. Laser Technol., 142, (2021) 107201.

[11] Yang Liao, Ya Cheng, Changning Liu, Jiangxin Song, Fei He, Yinglong Shen, Danping Chen, Zhizhan Xu, Zhichao Fan, Xunbin Wei, Koji Sugioka, and Katsumi Midorikawa: Lab Chip, 13, (2013) 1626.

[12] O.V. Andreeva, I.E. Obyknovennaya, E.R. Gavrilyuk, A.A. Paramonov, and A.P. Kushnarenko: J. Opt. Technol., 72, (2005) 916.

[13] M. Ams, G.D. Marshall, D.J. Spence, and M.J. Withford: Opt. Express, 13, (2005) 5676.

[14] L. Zhong, R.A. Zakoldaev, M.M. Sergeev, V.P. Veiko, and Z. Li: Opt. Quantum Electron., 52, (2020) 49

[15] A. Szameit and S. Nolte: J. Phys. B: At. Mol. Opt. Phys., 43, (2010) 163001.

[16] Y.M. Andreeva, M.M. Sergeev, R.A. Zakoldaev, U.E. Gabysheva, V.P. Veiko, E.B. Yakovlev, S.I. Kudryashov, P.A. Danilov, A.A. Ionin, F. Vocanson, T.E. Itina, T.V. Antropova, O.S. Medvedev: J. Laser Micro Nanoeng., 13, (2018) 193.

[17] A.Couairon, A.Mysyrowicz: Phys. Rep, 441, (2007) 47. 
[18] Yongfeng Ju, Yang Liao, Long Zhang, Yinglong Sheng, Qiang Zhang, Danping Chen, Ya Cheng, Zhizhan $\mathrm{Xu}$, Koji Sugioka, and Katsumi Midorikawa: Microfluid Nanofluid, 11, (2011) 111.

[19] Yang Liao, Yongfeng Ju, Long Zhang, Fei He, Qiang Zhang, Yinglong Shen, Danping Chen, Ya Cheng, Zhizhan $\mathrm{Xu}$, Koji Sugioka, and Katsumi Midorikawa: Opt. Lett., 35, (2010) 3225.

[20] S Nikumb, Q Chen, C Li, H Reshef, HY Zheng, H Qiu, D Low: Thin Solid Films, 477, (2005) 216.

(Received: July 8, 2021, Accepted: November 13, 2021) 